\section{Brustkrebs: Zweitlinie bleibt sekundär}

In der Zweitlinie wird beim Mammakarzinom häufig auf eine Kombination aus Vinorelbin und Capecitabin (NX) zurückgegriffen. Zuletzt hat dieses Regime auch überzeugend bei chemotherapienaiven Patientinnen gewirkt. Prospektiv untersuchten chinesische Forscher dies nun in der Erstlinie.

B eim metastasierten Mammakarzinom werden zunehmend Kombinationen von Anthrazyklinen und Taxanen verwendet. Das macht das Management von Rezidiven jedoch komplizierter - v.a. weil mit Anthrazyklinen vorbehandelte Patientinnen gemeinhin nicht mehr auf Anthrazykline ansprechen. Jüngst wurde allerdings gezeigt, dass Docetaxel/Capecitabin (TX) eine gute Zweitlinientherapie ist, wenn nach Anthrazyklinbehandlung Rezidive auftreten. Erste Erfolge gab es auch bereits mit NX in der Erstlinie.

Deshalb verglichen chinesische Kollegen beide Regimes prospektiv bei 206
Patientinnen mit metastasiertem Mammakarzinom: 104 Patientinnen erhielten randomisiert TX, $102 \mathrm{NX}$, alle außerdem eine Erhaltungstherapie mit Capecitabin. Primärer Endpunkt war das progressionsfreie Überleben (PFS).

Weil dieser früh erreicht war, wurde die Studie nach der Interimsanalyse abgebrochen. $\mathrm{Zu}$ dieser Zeit erhielten 48 Patientinnen in der TX-Gruppe (46,2\%) und 42 in der NX-Gruppe $(41,2 \%)$ die Erhaltungstherapie. Unter TX schienen im Vergleich zur NX-Gruppe das mediane PFS (8,4 vs. 7,1 Monate; Hazard Ratio [HR] 1,$65 ; \mathrm{p}=0,0026)$ und das mediane Gesamtüberleben (OS; 35,3 vs. 19,8
Monate; HR 1,48; $\mathrm{p}=0$,1349) günstiger $\mathrm{zu}$ sein. Allerdings erreichte letzterer Unterschied keine Signifikanz.

Postmenopausale Patientinnen $\geq 40$ Jahre mit viszeralen Metastasen profitierten eher von TX, was PFS und OS betraf. Ein positiver Hormonrezeptorstatus oder eine HER2-Expression beeinflussten das Überleben ebensowenig wie eine vorausgehende Taxantherapie.

Fazit: Die Behandlung des fortgeschrittenen Mammakarzinoms mit TX, gefolgt von einer Capecitabin-Erhaltungstherapie, führt offenbar zu einem längeren PFS als NX, selbst bei Patientinnen, die zuvor schon (neo-)adjuvant Taxane erhalten hatten.

Christina Berndt

Wang J et al. Capecitabine Combined With Docetaxel Versus Vinorelbine Followed by Capecitabine Maintenance Medication for First-Line Treatment of Patients With Advanced Breast Cancer: Phase 3 Randomized Trial. Cancer. 2015;121(19):3412-21.

\section{Mammakarzinom: Kardiale Toxizität unter Trastuzumab gering, aber nicht null}

In einer großen Studie wird noch einmal ein genauer Blick auf die kardiale Toxizität des Antikörpers Trastuzumab geworfen. Bei Patientinnen mit Risikofaktoren könnte es demnach wichtig sein, die Therapiedauer zu beschränken.

n der französischen PHARE-Studie wurden 3.380 Patientinnen mit Trastuzumab behandelt - jeweils die Hälfte über 12 bzw. 6 Monate. Während der Trastuzumab-Therapie sowie in den ersten zwei Jahren danach wurde alle $3 \mathrm{Mo}-$ nate ein kardiales Follow-up durchgeführt, auch wurde die linksventrikuläre Ejektionsfraktion (LVEF) mittels Echo-

kardiografie oder Radionuklid-Ventrikulo-

grafie (MUGAScan) bestimmt. Später erfolgten die Kontrolluntersuchungen

Kardiotoxizität unter Trastuzumab auf dem Prüfstand. nur noch alle 6 Monate. Primärer kardialer Endpunkt war eine Herzinsuffizienz (NYHA-Klasse III oder IV), sekundäre Endpunkte waren kardiale Ereignisse, kardiale Dysfunktionen (NYHA-Klasse I und II, LVEF-Abfall) und die Erholung der Herzfunktion.

Im Rahmen der Studie wurden im 12-Monats-Arm 14.055 LVEF-Messungen vorgenommen, im 6-Monats-Arm 13.218.Die Gesamtinzidenz einer Herzinsuffizienz betrug $0,65 \%(11 / 1.690)$ im 12 und $0,53 \%$ (9/1.690) im 6-Monats-Arm $(\mathrm{p}>0,05)$. Kardiale Dysfunktion trat bei $5,9 \%(100 / 1.690)$ bzw. 3,4\% (58/1.690) der Patientinnen auf $(\mathrm{p}=0,001)$. Bei der Mehrzahl der Patientinnen erholte sich die Herzfunktion allerdings; bleibende Folge erlitten 0,79\% (27/3.380). Mit Hilfe einer Subgruppenanalyse wurden vier besondere Risikofaktoren für kardiale
Ereignisse identifiziert: die sequenzielle Gabe von Trastuzumab und Chemotherapie (Subdistribution Hazard Ratio [SHR] 1,43), eine initiale LVEF von weniger als $55 \%$ (SHR 5,12), rechtsseitige Tumoren (SHR 1,36) sowie mehr als drei Zyklen einer Anthrazyklin-basierten Chemotherapie (SHR 1,71). Aus den drei zuletztgenannten Faktoren ließen sich mithilfe einer multivariaten Analyse drei Risikogruppen errechnen, innerhalb derer jeweils die Dauer der Trastuzumab-Gabe die Inzidenz der kardialen Ereignisse erhöhte.

Fazit: Die Studie bestätigt, dass die Inzidenz kardialer Ereignisse unter Trastuzumab niedrig, aber nicht null ist, wobei die Dauer der Therapie durchaus einen Einfluss hat. Die kardiale Toxizität ist aber zum Großteil reversibel. Patientinnen mit hohem kardialen Risiko sollten schon vor Beginn der Behandlung identifiziert werden; für sie kann eine kürzere Behandlung mit Trastuzumab eine Option sein.

Christina Berndt

Pivot $\mathrm{X}$ et al. Cardiac toxicity events in the PHARE trial, an adjuvant trastuzumab randomised phase III study. Eur J Cancer. 2015;51(13):1660-6. 